\title{
Manejo endovascular da síndrome de quebra-nozes: relato de caso
}

\author{
Endovascular management of nutcracker syndrome: case report \\ Manejo endovascular del síndrome del cascanueces: reporte de un caso
}

Thailena Silva Lima ${ }^{1 *}$, João Edison de Andrade Filho ${ }^{1}$, Frederico Augusto de Carvalho Linhares Filho ${ }^{1}$.

\section{RESUMO}

Objetivo: Descrever um caso diagnosticado de uma doença rara conhecida como Síndrome de Quebra-nozes (SQN) ou Nutcracker, tratado por via endovascular, apresentando eficácia satisfatória na técnica. Detalhamentos de Caso: Paciente do sexo feminino, 22 anos, apresentando dor pélvica crônica com irradiação para flanco esquerdo e episódio de hematúria. Realizou tomografia computadorizada que evidenciou compressão da veia renal esquerda, dilatação da veia gonadal esquerda e presença de varizes pélvicas bilaterais, tendo diagnóstico da doença. Foi submetida à angioplastia com stent em veia renal esquerda. Recebeu alta hospitalar com melhora dos sintomas. Durante seguimento, evoluiu com complicações pós-operatórias, sendo tratadas adequadamente. Realizou tomografia de controle mostrando perviedade do stent em veia renal esquerda. Está em acompanhamento ambulatorial com a cirurgia vascular e a urologia. Considerações finais: $O$ tratamento da doença é discutível e de acordo com a sintomatologia, embora a técnica endovascular com implante de stent tenha obtido excelentes resultados, sendo considerada segura, eficaz, menos invasiva e com menor morbimortalidade.

Palavras-chave: Síndrome do quebra-nozes, Dor pélvica, Hematúria, Stents.

\begin{abstract}
Objective: To describe a diagnosed case of a rare disease known as Nutcracker Syndrome (SQN) or Nutcracker, treated endovascularly, showing satisfactory effectiveness in the technique. Case Details: Female patient, 22 years old, presenting chronic pelvic pain with irradiation to the left flank and hematuria episode. Computed tomography scan showed compression of the left renal vein, dilation of the left gonadal vein and the presence of bilateral pelvic varices, diagnosing the disease. She underwent angioplasty with a left renal vein stent. He was discharged from hospital with improvement of symptoms. During follow-up, she evolved with postoperative complications, being treated appropriately. Control tomography was performed showing patency of the stent in the left renal vein. He is being followed up on an outpatient basis with vascular surgery and urology. Final considerations: The treatment of the disease is debatable and according to the symptoms, although the endovascular technique with stent implantation has obtained excellent results, being considered safe, effective, less invasive and with less morbidity and mortality.
\end{abstract}

Keywords: Renal nutcracker syndrome, Pelvic pain, Hematuria, Stents.

\section{RESUMEN}

Objetivo: Para describir un caso diagnosticado de una enfermedad rara conocida como Síndrome del Cascanueces (SQN) o Cascanueces, tratada endovascularmente, mostrando una efectividad satisfactoria en la técnica. Detalles del Caso: Paciente de sexo femenino, 22 años, que presenta dolor pélvico crónico con irradiación en flanco izquierdo y episodio de hematuria. La tomografía computarizada mostró compresión de

\footnotetext{
${ }^{1}$ Hospital Universitário Walter Cantídio / UFC, Fortaleza - CE. *E-mail: thailenasilva@gmail.com
} 
la vena renal izquierda, dilatación de la vena gonadal izquierda y presencia de varices pélvicas bilaterales, diagnosticando la enfermedad. Fue sometida a angioplastia con stent en vena renal izquierda. Fue dado de alta del hospital con mejoría de los síntomas. Durante el seguimiento evolucionó con complicaciones postoperatorias, siendo tratada adecuadamente. Se realiza tomografía de control que muestra la permeabilidad del stent en la vena renal izquierda. Está en seguimiento de forma ambulatoria con cirugía vascular y urología. Consideraciones finales: El tratamiento de la enfermedad es discutible y acorde a la sintomatología, aunque la técnica endovascular con implante de stent ha obtenido excelentes resultados, considerándose segura, eficaz, menos invasiva y con menor morbimortalidad.

Palabras clave: Síndrome de cascanueces renal, Dolor pélvico, Hematuria, Stents.

\section{INTRODUÇÃO}

A Síndrome de Quebra-nozes (SQN) ou Nutcracker é uma doença, que acomete mulheres entre 20 e 40 anos. De diagnóstico difícil e geralmente tardio, resulta de um conjunto de sinais e sintomas secundários a compressão da veia renal esquerda (VRE), mais comumente situada entre a artéria mesentérica superior (AMS) e a aorta (AA). O ângulo aortomesentérico normal é de $90^{\circ}$; porém, quando é agudo, ocorre a compressão da VRE anteriormente, na maioria dos casos. Quando a veia renal está situada retroaórtica, a compressão acontece posteriormente pela aorta e pelo corpo vertebral. A prevalência e incidência da patologia ainda permanecem desconhecidas por ser rara e apresentar variabilidade dos sintomas, sendo frequentes a dor abdominal e hematúria, podendo ser agravados com a atividade física intensa (JÚNIOR JRC, et al., 2013; DE MACEDO GL, et al., 2018; BELCZAK SQ, et al., 2020).

Importante causa de dor pélvica crônica que traz impacto na qualidade de vida e custos para os serviços de saúde uma vez que a doença, na maioria das vezes, acaba sendo subdiagnosticada e subvalorizada. A Síndrome Nutcracker pode estar relacionada à nefroptose ou escassez de gordura retroperitoneal, acarretando um alongamento da veia renal e uma diminuição do ângulo aortomesentérico, respectivamente. A hipertensão da veia renal esquerda ocasiona refluxo venoso, que resulta varizes pélvicas, sendo responsável por um quadro clínico de dor em flanco esquerdo e dor pélvica crônica, apresentando graus variados de dor e associando-se à congestão pélvica nas mulheres e varicocele nos homens (AGLE CG, et al., 2019; TEIXEIRA EL, et al., 2016; MARQUES GL, et al., 2012).

O diagnóstico é feito através de uma anamnese detalhada, exame físico rigoroso e com base na exclusão de outras causas mais prevalentes, usando métodos de imagem como o doppler, angiotomografia e venografia retrógrada. Este último é o exame padrão ouro, embora invasivo, e o mais informativo, realizado em pacientes com sintomas graves. A cistoscopia é útil para identificar hematúria de origem ureteral esquerda (DE MACEDO GL, et al., 2019; MACHADO M, et al., 2018; GULLEROGLU K, et al., 2014).

A investigação diagnóstica inicia-se com o exame ecodoppler, por ser de fácil acesso e permitir o estudo dinâmico do fluxo venoso. $O$ gradiente venoso entre a veia renal e a cava inferior $\geq 3 \mathrm{mmHg}$, o Doppler com aumento $>5$ vezes na velocidade do fluxo na passagem pela artéria mesentérica superior em relação ao hilo renal e a angiotomografia ou angiorressonância com ângulo < 45 são critérios sugestivos para o diagnóstico da Síndrome de Nutcracker (DE ALMEIDA R, et al., 2010; GALEGO GN, et al., 2015).

O tratamento da patologia é discutível, de acordo com a gravidade dos sintomas e sua reversibilidade esperada em relação à idade do paciente e ao estágio da síndrome. Nos casos de pacientes com sintomas leves e toleráveis, é recomendado o manejo conservador com analgesia, suplementação de ferro e antiagregação plaquetária se necessária. No entanto, a cirurgia está indicada para sintomas graves e medidas conservadoras ineficazes após 24 meses em pacientes com idade inferior a 18 anos e após 6 meses em adultos. A abordagem endovascular pode ser uma opção terapêutica menos invasiva, e eficaz para solução do problema através da colocação de stent, realização de angioplastia com balão ou embolização (CALADO R, et al., 2011; ANANTHAN K, et al., 2017; GULLEROGLU K, et al., 2014).

O presente trabalho tem por objetivo descrever um caso diagnosticado de Síndrome de Quebra-nozes (SQN), tratado por via endovascular, apresentando eficácia satisfatória na técnica. 


\section{DETALHAMENTO DO CASO}

Paciente do sexo feminino, 22 anos, branca, procedente de Fortaleza, apresentando dor pélvica com irradiação para flanco esquerdo há cerca de 1 ano. Procurou atendimento médico, sendo solicitado ultrassom (US) transvaginal que mostrou presença de varizes pélvicas e US abdominal em fevereiro/2019 que evidenciou nefrolitíase à esquerda. Procurou o serviço de urologia e foi optado por conduta conservadora, fazendo uso de analgésicos e anticoncepcional combinado contínuo. Porém, evoluiu com persistência de dor em flanco esquerdo, disúria, esforço miccional e sensação de esvaziamento incompleto.

Realizou uma tomografia computadorizada (TC) sem contraste em maio/2019 mostrando ausência de litíase renal e sinais de compressão da veia renal esquerda entre a aorta e a artéria mesentérica superior com ectasia da veia gonadal esquerda de aspecto varicoso compatível com Síndrome de Nutcracker.

Paciente foi encaminhada ao ambulatório de cirurgia vascular de um hospital público, nível terciário, do município de Fortaleza/CE. Apresentava intensa dor em flanco esquerdo que não cessava com analgésicos potentes.

Foi internada para analgesia endovenosa e realização de tomografia com contraste (julho/2019) que mostrou compressão da veia renal esquerda entre a artéria mesentérica superior e a aorta, dilatação da veia gonadal esquerda e presença de varizes pélvicas bilaterais, tendo como diagnóstico a Síndrome de quebranozes.

Durante internamento, apresentou hematúria macroscópica. Após medidas clínicas, evoluiu com melhora dos sintomas, recebendo alta hospitalar para aguardar chegada do material para tratamento endovascular (Figura 1).

Figura 1 - TC realizada antes do tratamento mostrando a compressão da veia renal esquerda (apontada pela seta) entre a aorta e a artéria mesentérica superior.

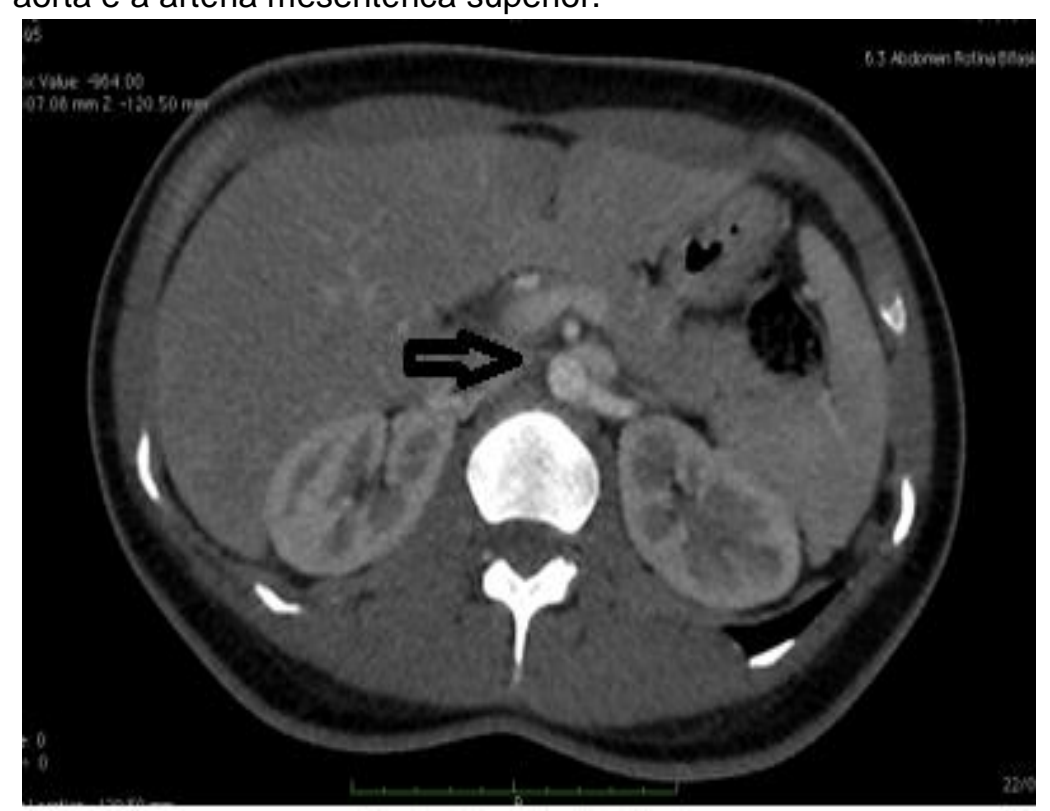

Fonte: Lima TS, et al., 2020.

Em outubro/2019, referiu piora do quadro álgico. O procedimento endovascular foi realizado sob anestesia geral, sendo puncionada a veia femoral direita, introduzida uma bainha curta $6 \mathrm{~F}$ e feito a cateterização seletiva da veia renal esquerda sob guia rígido, com realização de flebografia pré-procedimento mostrando compressão da veia renal esquerda, dilatação significativa de veia ovariana e presença de circulação colateral com enchimento para veia gonadal esquerda. 
Procedeu-se com a passagem de cateter balão over-the-wire $5 \mathrm{~mm} \times 100 \mathrm{~mm}$ no ponto de pinçamento da veia renal esquerda e angioplastia do mesmo. Ocorreu a troca do introdutor pelo de bainha longa 10F. Foi realizado o implante de stent autoexpansível Wallstent ${ }^{\circledR}-14 \mathrm{~mm} \times 60 \mathrm{~mm}$ em veia renal esquerda com o cuidado de manter área de fixação proximal e distal para evitar migração para a cava (Figura 2).

Figura 2 - Flebografia pré-angioplastia.

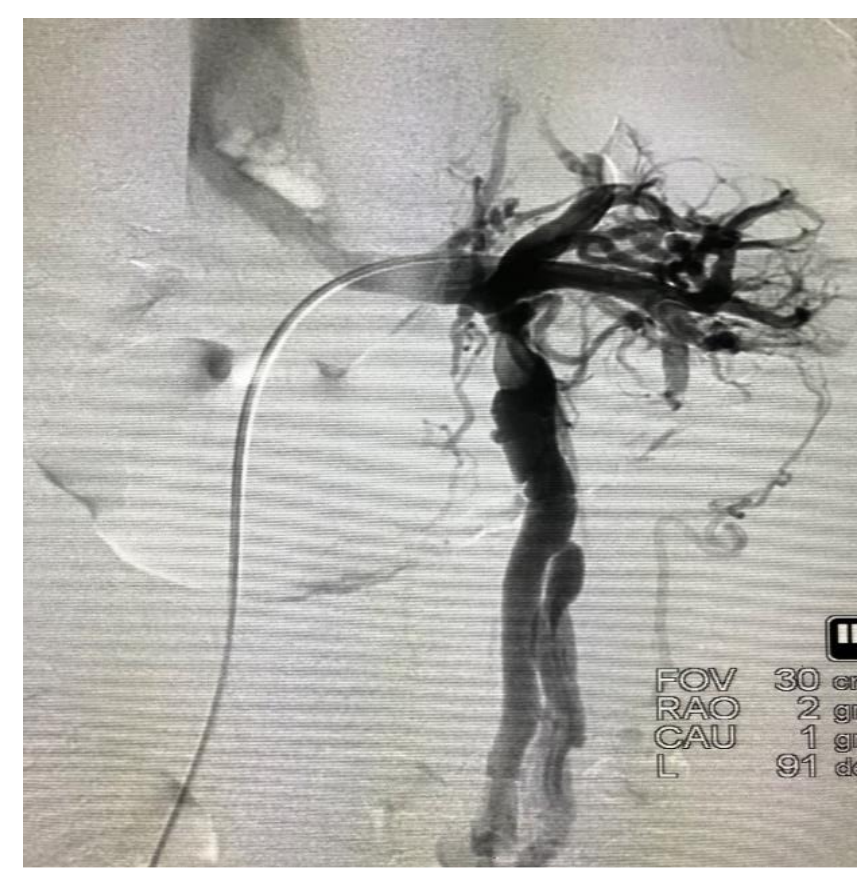

Fonte: Lima TS, et al., 2020.

Foi puncionada a veia femoral esquerda com introdução de bainha curta $5 \mathrm{~F}$ para flebografia de controle, apresentando correção satisfatória da compressão da veia renal esquerda. Realizada a retirada do sistema e a compressão dos sítios de punção (Figura 3).

Figura 3 - Flebografia de controle mostrando stent (destacado pela seta) implantado em VRE.

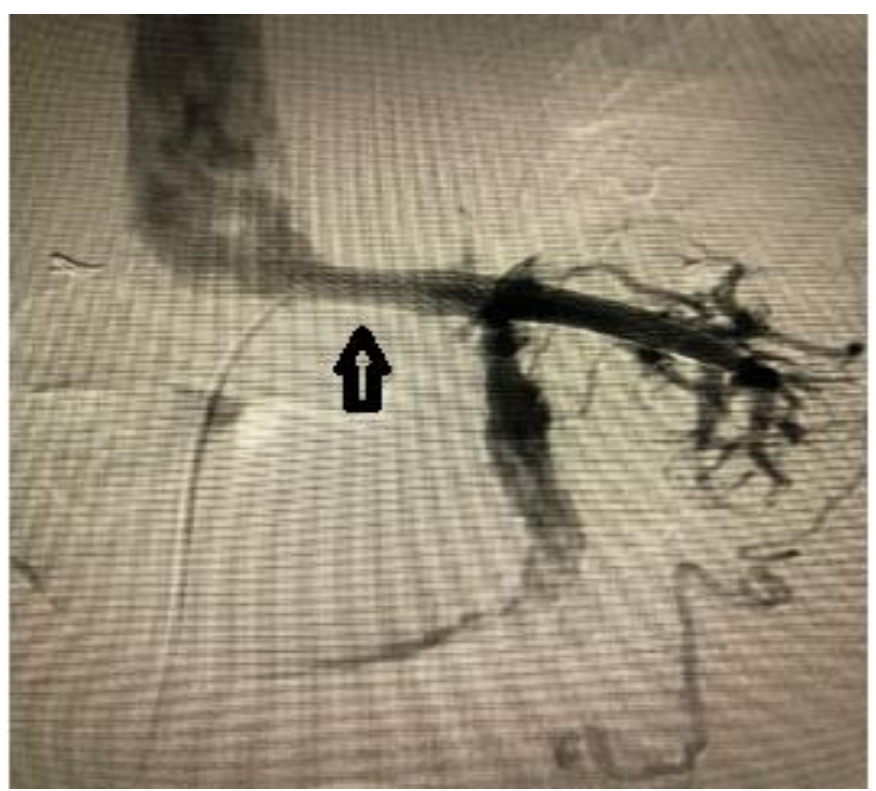

Fonte: Lima TS, et al., 2020. 
A paciente evoluiu no pós-operatório imediato com dor lombar, náuseas e vômitos. Após medidas clínicas, houve remissão do quadro. Recebeu alta hospitalar após 4 dias da internação com prescrição de AAS 100 $\mathrm{mg} / \mathrm{dia}$ e clopidogrel $75 \mathrm{mg} / \mathrm{dia}$. Após 1 mês do procedimento, na consulta ambulatorial, relatou sangramento transvaginal e dor em flanco esquerdo, sendo encaminhada para ginecologista e solicitados exames laboratoriais, sumário de urina, US transvaginal e de vias urinárias.

AAS e clopidogrel foram suspensos. Paciente retornou uma semana depois relatando parada do sangramento, persistência de dor e presença de disúria. Trouxe US de vias urinárias mostrando perviedade do stent em veia renal esquerda.

US transvaginal não mostrava alterações. Foi ao ginecologista que descartou causas ginecológicas acerca do sangramento. Sumário de urina mostrava leucocitúria, e hemoglobina com níveis dentro da normalidade. Iniciada antibioticoterapia oral, porém, a mesma retornou à consulta com dor intensa em flanco esquerdo e ao exame físico apresentava sinal de Giordano positivo.

Internou-se para investigação diagnóstica. Durante internamento, relatou hematúria macroscópica, sendo diagnosticada pielonefrite. Fez uso de antibioticoterapia venosa. Realizou nova tomografia em novembro/2019 que evidenciou descompressão adequada da veia renal esquerda, perviedade do stent e trombose de veia gonadal esquerda.

Solicitado parecer da urologia que orientou tratamento conservador. Paciente evoluiu com cessação da hematúria e melhora da dor, recebendo alta após 16 dias de internação. No momento, encontra-se em acompanhamento ambulatorial com a cirurgia vascular e a urologia (Figura 4).

Figura 4 - Tomografia de controle após tratamento mostrando a perviedade do stent (conforme aponta a seta).

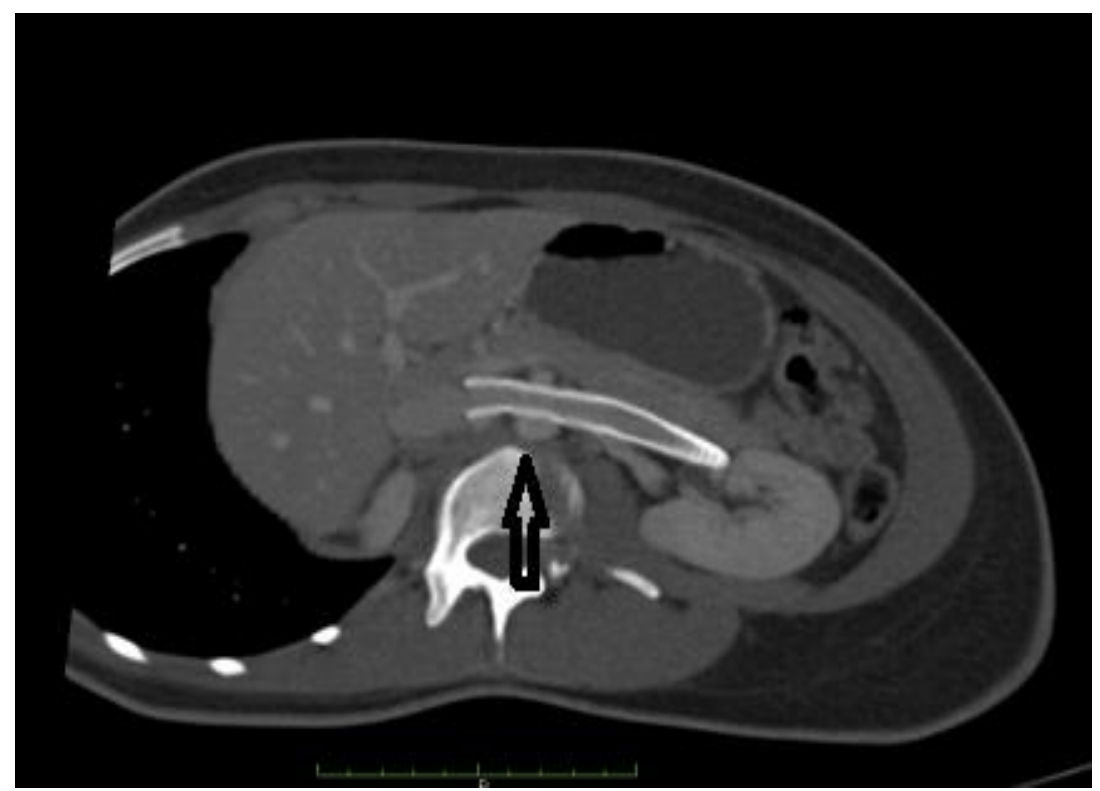

Fonte: Lima TS, et al., 2020.

\section{DISCUSSÃO}

Em 1950, El Sadr descreveu pela primeira vez a síndrome de compressão da veia renal esquerda pela aorta e artéria mesentérica superior, sendo denominada, em 1972, por De Schepper, como "síndrome de Quebra-nozes" (Nutcracker). Tal patologia é diagnosticada quando ocorre redução do calibre da VRE maior que $50 \%$, ao cruzar a aorta abdominal. Esta situação provoca uma variabilidade de manifestações clínicas desde o estado assintomático até sintomas graves como hematúria maciça associada à anemia severa (JÚNIOR JRC, et al., 2013; MARQUES GL, et al., 2012). 
O tratamento ainda continua controverso e varia de acordo com a gravidade dos sintomas do paciente. Tem como opções: manejo conservador, cirurgia convencional, incluindo desde nefropexia, bypass da veia renal, transposição da VRE, transposição da AMS, autotransplante renal, derivação renocaval, bypass gonadocaval até nefrectomia, e a terapêutica endovascular que envolve a colocação de stent externo ou intravascular e embolização. O tratamento clínico é indicado nos casos poucos sintomáticos e toleráveis. Já o cirúrgico e endovascular, são recomendados nos casos com sintomas severos. A intervenção e escolha do método devem ser adaptadas ao paciente individual e suas condições particulares (DENCHEV B, et al., 2018; MACHADO M, et al., 2018; BELCZAK SQ, et al., 2020).

A cirurgia, nos casos graves, se faz necessária para evitar a piora da função renal bem como a dilatação permanente da veia gonadal e trombose da veia renal. A transposição da veia renal foi realizada pela primeira vez em 1982 e se mostrou eficaz. É a cirurgia mais frequente e tem sido padrão-ouro para tratamento da doença. Alguns pacientes, apesar do sucesso desse procedimento, mantêm hematúria e necessitam efetuar uma intervenção cirúrgica complementar. O autotransplante renal é a técnica mais invasiva e apresenta riscos como tempo de isquemia renal, anastomoses de artéria renal e ureter e necessidade de grande exposição cirúrgica. A derivação renocaval é um procedimento de experiência limitada e resultados a longo prazo. A nefrectomia é o mais radical, sendo indicada em casos refratários a diferentes abordagens terapêuticas, os métodos cirúrgicos convencionais associam-se a uma maior morbidade, quando comparados aos métodos menos invasivos, devido ao longo período de congestão renal, à necessidade de anastomoses adicionais e à extensa dissecção (DE MACEDO GL, et al., 2018; AGLE CG, et al., 2019).

O manejo endovascular tornou-se popular nos últimos anos como uma alternativa minimamente invasiva para o tratamento de doenças obstrutivas do sistema venoso. Segundo a literatura, foram relatadas taxas de patência primária de 66\% em 3 anos e taxas de complicações menores que 2\% (MACHADO M, et al., 2017). Essa abordagem vem substituindo progressivamente a cirurgia convencional para tratamento da síndrome de Quebra-nozes por apresentar eficácia satisfatória na técnica e menor morbimortalidade. Além disso, traz excelente benefício na evolução clínica do paciente (MACHADO M, et al., 2018; WANG X, et al., 2012).

O implante de stent endovascular é usualmente preferível à cirurgia aberta, pois este último método apresenta mais complicações e necessidade de extensa dissecção. Além disso, o tratamento endovascular permite a realização simultânea de embolização da veia gonadal e/ou esclerose com polidocanol diretamente nas varizes pélvicas durante o procedimento caso sejam necessárias. O stent ideal tem que ter alta força radial, boa conformabilidade e pouco encolhimento no comprimento. Os mais frequentemente utilizados são autoexpansíveis como o Wallstent, e a recomendação é de que seja usado um dispositivo de 6 ou $8 \mathrm{~cm}$ de comprimento, posicionando-o na primeira porção da veia renal esquerda (AGLE CG, et al., 2019; DE MACEDO GL, et al., 2018). Após o tratamento, os pacientes necessitam usar medicamento anticoagulante e antiagregante plaquetário por 2 a 3 meses. O esquema recomendado consiste em 3 dias de heparina de baixo peso molecular, 30 dias de clopidogrel e 3 meses de aspirina (DE MACEDO GL, et al., 2018).

O método endovascular tem apresentado excelentes resultados no tratamento de doenças de obstrução venosa e vem sendo indicado mais frequentemente, embora ainda não existam estudos suficientes na literatura mostrando acompanhamento a longo prazo de pacientes tratados, tornando-o ainda limitado. As técnicas mais utilizadas são a colocação do stent e embolização da veia gonadal esquerda. Apesar de ter baixos índices, suas complicações incluem principalmente migração do stent, fratura e oclusão venosa, exigindo cautela e experiência por parte do cirurgião vascular. Espera-se que, no futuro, haja mais conhecimentos teóricos que comprovem a segurança e eficácia da terapêutica para que essa abordagem seja considerada primeira escolha no tratamento de portadores da Síndrome de Nutcracker (DE MACEDO GL, et al., 2018; WANG X, et al., 2012; JÚNIOR JRC, et al., 2013).

Contudo, a técnica endovascular é uma alternativa excelente, eficaz e segura, que tem sido cada vez mais utilizada, embora permaneça uma possibilidade de desenvolver hiperplasia fibromuscular da íntima e levar a trombose venosa (FENG KK, et al., 2013; CORREIA T, et al., 2007; AVGERINUS AD, et al., 2013).

Conclui-se que a Síndrome de Quebra-nozes é uma doença rara e subdiagnosticada, sendo importante o conhecimento da patologia para a realização do diagnóstico precoce e o manejo adequado do paciente.

REAS/EJCH | Vol.12(12) | e5112 | DOI: https://doi.org/10.25248/reas.e5112.2020 Página 6 de 7 
Dentre os métodos de tratamento, o endovascular tem se mostrado seguro, eficaz, menos invasivo, com menor risco de complicações e menor morbimortalidade, contudo, ainda não há consenso na literatura acerca dessa terapêutica na Síndrome de Nutcracker, sendo necessário trabalhos científicos com acompanhamento a longo prazo.

\section{AGRADECIMENTOS E FINANCIAMENTO}

Agradeço a equipe do serviço de cirurgia vascular do hospital, onde fiz residência médica, pelo aprendizado, pela dedicação com os pacientes e pelo esforço na resolução de casos complexos.

\section{REFERÊNCIAS}

1. AGLE CG, et al. Tratamento endovascular da síndrome de Quebra-nozes: relato de caso. Jornal Vascular Brasileiro, 2019;18(1): 1-6.

2. AVGERINUS AD, et al. Surgical and endovascular interventions for nutcracker syndrome. Semin Vasc Surg. 2013; 26(4): 170-7.

3. ANANTHAN K, et al. Nutcracker Syndrome: An Update on Current Diagnostic Criteria and Management Guidelines. European Journal of Vascular and Endovascular Surgery, 2017; 53(6): 886-894.

4. BELCZAK SQ, et al. Tratamento endovascular da síndrome do quebra-nozes em adolescente: relato de caso. Jornal Vascular Brasileiro, 2020;19 (1): 1-6.

5. CALADO R, et al. SÍNDROME DE NUTCRACKER Hematúria sem diagnóstico? Acta Médica Portuguesa, 2011; 24(S3): 695-698.

6. CORREIA T, et al. Síndrome de Quebra-nozes: A propósito de um caso clínico. Acta Urologica. 2007;24(4):49-52.

7. DE ALMEIDA R, et al. Síndrome de Nutcracker. Relato de caso. Rev Bras Clin Med 2010;8(3):283-5.

8. DE MACEDO GL, et al. Diagnóstico e tratamento da síndrome de quebra-nozes (nutcracker): revisão dos últimos 10 anos. Jornal Vascular Brasileiro, 2018;17(3):220-228.

9. DE MACEDO GL, et al. Revascularização venosa via transposição de veia gonadal esquerda em síndrome de nutcracker posterior: relato de caso. Jornal Vascular Brasileiro, 2019;18(1): 1-4.

10. DENCHEV B, et al. Surgical Treatment of a Patient with Nutcracker Syndrome via Transposition of the Left Renal Vein. EJVES Short Reports, 2018; 41(1): 10-12.

11. FENG KK, et al. Endovascular stenting for nutcracker syndrome. J Chin Med Assoc. 2013;76(6):350-3.

12. FERREIRA M, et al. Dor pélvica crônica: o papel da síndrome do quebra-nozes. Jornal Vascular Brasileiro, 2008; 7(1): 76-79.

13. GALEGO GN, et al. Síndrome da Congestão Venosa Pélvica e resultados do tratamento endovascular: série de casos. Jornal Vascular Brasileiro, 2015; 14(3):262-266.

14. GULLEROGLU K, et al. Nutcracker syndrome. World J Nephrol, 2014; 3(4): 277-281.

15. JÚNIOR JRC, et al. Tratamento endovascular da Síndrome de Quebra-nozes (Nutcracker). Jornal Vascular Brasileiro, 2013; 12(3): 247-251.

16. MACHADO M, et al. Sindrome de May-Thurner associado a um sindrome de Nutcracker: caso clinico e revisão da literatura. Angiologia e Cirurgia Vascular, 2017; 13(2): 52-56.

17. MACHADO M, et al. Síndrome de Nutcracker: Uma patologia rara e subdiagnosticada? A nossa eexperiência, revisão da literatura e proposta de uma nova classificação anatômica da Síndrome de Nutcracker. Angiologia e Cirurgia Vascular, 2018;14(2): 26-34.

18. MARQUES GL, et al. Síndrome de quebra-nozes (nutcracker) em uma mulher jovem em investigação por quadro de hematúria recorrente: um relato de caso. Jornal Brasileiro de Nefrologia, 2012;34(2):195-198.

19. TEIXEIRA EL, et al. Síndrome de Nutcracker: Um Diagnóstico a Evocar. Acta Pediátrica Portuguesa, 2016; 47(1):182186.

20. WANG X, et al. Results of endovascular treatment for patients with nutcracker syndrome. Journal of Vascular Surgery, 2012; 56(1): 142-148. 